\title{
Tailored treatment for postpartum depression
}

$\mathrm{H}$ aving suffered from depression following the birth of two children and throughout her life, Amy Martin was surprised when storm clouds didn't immediately gather after the delivery of her third. Recovering in hospital from an emergency cesarean section, Martin felt okay - "no one feels great after childbirth," she quips - and hoped she'd eluded the baby blues.

"Then I went home, and it was like I hit a brick wall," she says. "I wasn't taking care of myself, wasn't eating, wasn't sleeping, was kind of catatonic, and I was having these awful, terrible thoughts, panic attacks, and was afraid I would hurt the baby or myself. It was like nothing I had experienced before."

Specialty inpatient clinics for women with severe postpartum depression or psychosis have been operating in some European countries for more than 50 years. But earlier this month, the University of North Carolina at Chapel Hill opened what is believed to be the first such clinic in North America when it officially unveiled its Perinatal Psychiatry Inpatient Unit.

Everything in the unit is tailored to women with postpartum depression, says Dr. Samantha Meltzer-Brody, director of the university's Center for Women's Mood Disorders. The 24/7 service boasts up to five beds, as well as breast pumps, bassinets, rocking chairs and handheld biofeedback devices that help patients monitor their breathing and heart rates. It also allows extended visiting hours for infants to help moms establish routines, and offers an intensive range of therapies, from medication management, mother-infant attachment therapy and partner-assisted therapy, to mother-baby yoga, time management and coping skills, and mental health education.

"Some 10 to $15 \%$ of new moms will experience postpartum depression, and while outpatient services, support groups and crisis hotlines are effective for the majority, some $5 \%$ of

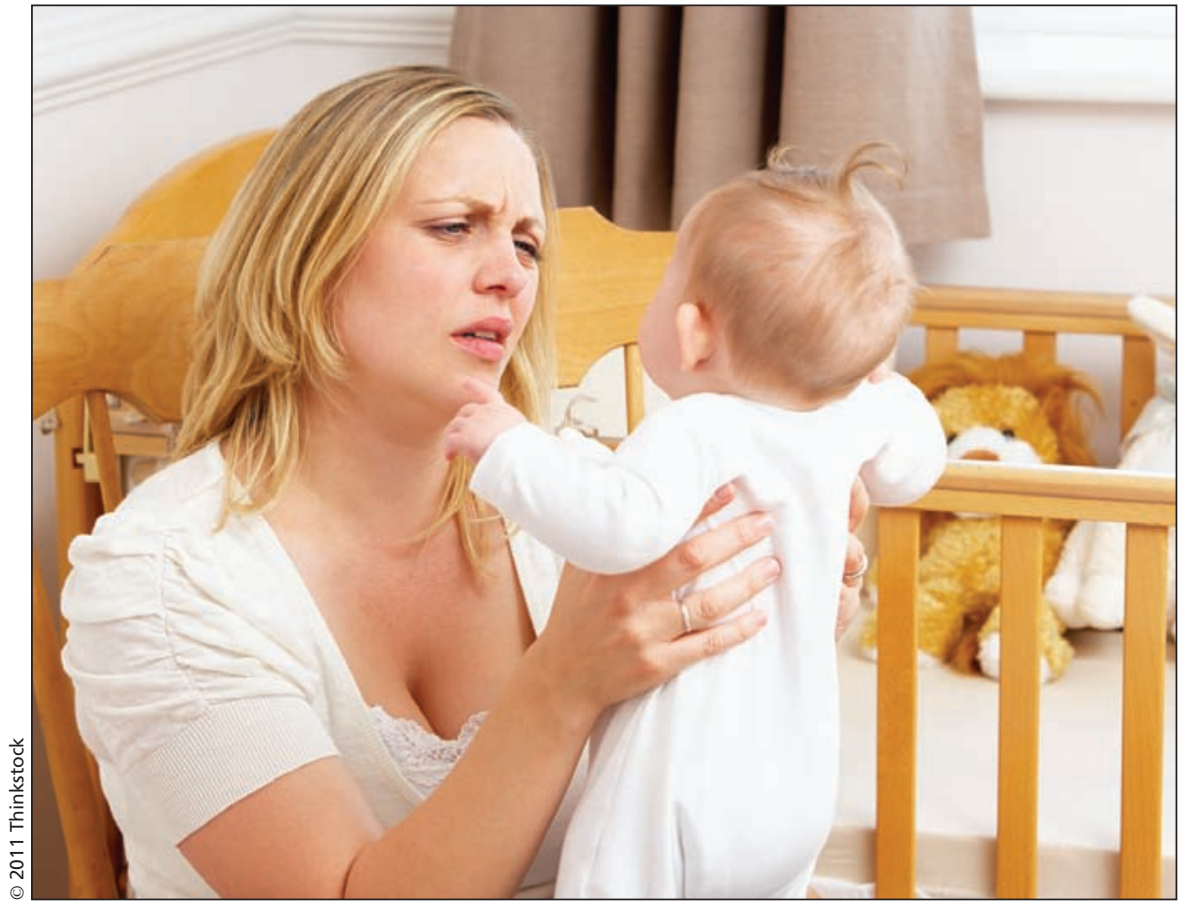

An estimated $10 \%-15 \%$ of new moms experience postpartum depression.

that group will experience symptoms so severe they require hospitalization," says Meltzer-Brody.

That typically meant admittance to a hospital's general psychiatric unit, most of which aren't equipped to accommodate crucial mother-infant interaction and bonding therapies, she explains. "There are two parties involved in postpartum depression, and you can't adequately treat mothers without having the baby involved in the care."

Fear of being "labelled crazy," and stigma surrounding psychiatric wards, can dissuade women from seeking care, says Martin, who participated in the pilot of the clinic that was conducted at the hospital's general psychiatry unit. "Women with postpartum depression require so much different care than other people with different mental illnesses, and it's important to have that space where women feel safe and are able to have their babies with them. I was apprehensive about being a mental patient in a psychiatric ward. I didn't want people to think I was nuts, but at the same time, because of my experiences with postpartum depression in the past, I was at the point where I knew I needed help."

University of North Carolina Health Care has embedded psychiatric nurse practitioners in its obstetrics and pediatrics wards in a bid to reach those women who are uncomfortable about directly approaching psychiatric clinics for assessment.

"Anything related to mental health still has stigma, certainly, but attitudes about and understanding of mental health have really transformed in recent years," Meltzer-Brody says. "Not least because the American College of Obstetricians and Gynecologists has really embraced it as a priority in the last few years. It really took people starting to research in this area to have a compelling story of the prevalence, the potential morbidity and mortality, that suicide is the number one cause of maternal death, and then celebrities like Brooke Shields started to come forward with their experiences." 
But a mere five inpatient beds in North Carolina to serve an entire country is far from adequate, Meltzer-Brody says. Screening remains inconsistent in much of the country and there's a lack of awareness about available resources. "Women are suffering unnecessarily, and their experiences vary from moderate symptoms to women killing themselves, or the rarer cases of women who have psychotic symptoms and kill their babies for the want of that care," she explains.
Postpartum psychosis occurs in 1 to 2 out of every 1000 deliveries, or approximately $0.1 \%$ of births, and is associated with a $5 \%$ infanticide or suicide rate, according to advocacy and support group Postpartum Support International (www.postpartum.net /Get-the-Facts/Depression-During-Preg nancy-Postpartum.aspx).

The clinic is expected to become a model for other facilities across North America. Since first being piloted in 2008 , the ward has had a wait list.
Postpartum depression advocates hope the program sparks changes in Canada. Although there are inpatient programs for reproductive mental health scattered across the country, there's "nothing that resembles what they're doing in North Carolina," says Gina Wong-Wylie, a registered psychologist in Edmonton, Alberta, and coordinator for Postpartum Support International Canada. — Lauren Vogel, CMAJ

CMAJ 2011. DOI:10.1503/cmaj.109-4003 